\title{
A SPACE-FRACTIONAL MONODOMAIN MODEL FOR CARDIAC ELECTROPHYSIOLOGY COMBINING ANISOTROPY AND HETEROGENEITY ON REALISTIC GEOMETRIES
}

\author{
N. CUSIMANO AND L. GERARDO-GIORDA
}

\begin{abstract}
Classical models of electrophysiology do not typically account for the effects of high structural heterogeneity in the spatio-temporal description of excitation waves propagation. We consider a modification of the Monodomain model obtained by replacing the diffusive term of the classical formulation with a fractional power of the operator, defined in the spectral sense. The resulting nonlocal model describes different levels of tissue heterogeneity as the fractional exponent is varied. The numerical method for the solution of the fractional Monodomain relies on an integral representation of the nonlocal operator combined with a finite element discretisation in space, allowing to handle in a natural way bounded domains in more than one spatial dimension. Numerical tests in two spatial dimensions illustrate the features of the model. Activation times, action potential duration and its dispersion throughout the domain are studied as a function of the fractional parameter: the expected peculiar behaviour driven by tissue heterogeneities is recovered.
\end{abstract}

Keywords: Cardiac electrophysiology; Fractional operators; Finite elements

\section{INTRODUCTION}

Heart electrophysiology is the subject of a vast interdisciplinary literature, from medical sciences to bio-engineering, physiology, chemistry and physics, where mathematical modelling and numerical simulations have been playing a crucial role for more than 20 years. The electrical activity of the heart as a whole is characterized by a complex multiscale structure, ranging from the microscopic activity of ion channels in the cellular membrane to the macroscopic properties of the anisotropic propagation of the excitation and recovery fronts in the whole heart.

If modern imaging techniques, such as high-resolution magnetic resonance imaging (MRI), allow high level of accuracy in the description of both the microstructure of the tissue and the global anatomy of the organ, most current mathematical models (the Bidomain and Monodomain equations, [1]) are still based on a formalism whose derivation is based on significant simplifying assumptions of the spatial domain complexity. From the anatomical standpoint, the heart features a complex structural organization. Elongated muscle cells form a network of branching and merging fibres that in ventricular walls exhibit a rotation of more than 100 degrees transmurally. In addition, cardiac walls contain other cell populations, such as fibroblasts, and structures like blood vessel at scales ranging from a few $\mu \mathrm{m}$ to several $\mathrm{mm}$. Although some models are currently including a mesoscale structure, such as the Purkinje tree in the ventricles $([2,3])$, the microscopic complexity of the cardiac wall has been largely ignored in whole-heart models, mainly because these small structures remain below the attainable size of discretisation grids [4], and incorporating effects of microstructures in correspondence with a much coarser discretisation grid is extremely challenging.

Mathematical models of electrical propagation in excitable media are typically derived via homogenisation in a periodic spatial arrangement of cardiomyocytes, with linear gap junctions. Such paradigm is intrinsically limited, as it is based on the assumption that microscopic inhomogeneities 
in the medium have a negligible effect on the transport phenomena observed at the macroscopic scale. In highly heterogeneous structures, such as cardiac or neural tissue, where there is no clear separation of scales, this hypothesis is questionable. Recent developments prompt a reconsideration of the level at which inhomogeneities should be included. In the first place, imaging resolution is rapidly increasing. In addition, there is a growing awareness of the importance of structural abnormalities in a wide range of cardiac diseases and the progressive remodelling that occurs during such diseases. In cardiac MRI, a recent work by Bueno-Orovio and colleagues [14] established a connection between myocardial microstructure and the experimentally observed anomalous diffusion of water molecules in the tissue. Moreover, experimental data of cardiac electrophysiological dynamics highlight peculiar features, such as wide action potential foot [6] and marked dispersion of Action Potential Duration (APD) [7], that are not captured by standard models, neither Bidomain nor Monodomain. As numerical modelling plays an increasing role in the development of knowledge in cardiac electrophysiology, these limitations hinder the reliability of the models themselves, as well as their viability to explore in depth complex pathological mechanisms. Alternative modelling strategies are thus needed to provide additional insight into the effect produced by structural heterogeneity.

In the last few decades, mathematical models involving differential operators of non-integer order have been considered in a variety of disciplines (such as physics, engineering, chemistry, rheology $[8,9,10,11,12,13])$ with the aim of reproducing transport phenomena whose characteristics significantly deviate from the classical Markovian and Gaussian features, typical of standard diffusion models. Although the interest in fractional operators linked to practical applications is increasingly growing, the successful implementations of fractional models to model real life phenomena are still scarce.

To the best of our knowledge, the work by Bueno-Orovio et al. [5] is the first example in which a space-fractional mathematical model of cardiac electrophysiology is studied. This is done by replacing the classical diffusive term in the model with a fractional power of the Laplacian operator which introduces nonlocality effects on the spatio-temporal propagation of the electrical wave. The biophysical justification given by the authors of [5] for the use of the fractional Laplacian operator in this particular application is based on potential theory and the effect produced on the electrical field by the various inhomogeneities present on a variety of length scales in biological tissue. This connection to potential theory allowed the authors of [5] to show that a fractional model can be interpreted as a smooth transition from the case of a perfectly homogeneous medium to a domain with increasing degree of heterogeneity as the order of the fractional operator decreases. Although capturing peculiar features of action potential propagation in heterogeneous media, and showing good agreement with experimental data, their numerical simulations are restricted to one-dimensional intervals.

The numerical method implemented in [5] exploits explicit knowledge of the eigenpairs of the Laplacian operator coupled to the desired boundary conditions on a one-dimensional interval. If the extension of this methodology to two and three dimensional cartesian domains is pretty straightforward, its application to real geometries is not practical, as eigenvalues and eigenfunctions are not known analytically in these cases. An efficient method for approximating the eigendecomposition of the Laplacian on more general domains has been recently proposed in [15]. Rather than directly computing the eigenpairs of the Laplacian, together with our collaborators we followed in [16] an alternative strategy to approximate the action of the nonlocal operator on a given function, exploiting the heat-semigroup formulation of the fractional operator in order to handle both general domains and general boundary conditions. Halfway between the two approaches, Burrage et al. [17] analysed in detail three methods for the solution of fractional-in-space reaction-diffusion equations involving fractional powers of a suitable matrix linked to the finite element mass and 
stiffness matrices of the classical heat equation, coupled to the desired boundary conditions (see further details in Section 3), perhaps not exploiting to the fullest the flexibility of their approach. In fact, they worked exclusively with fractional modifications of reaction-diffusion problems involving $(-\Delta)^{s}$, but the same strategies can be used for evolution equations involving more general fractional powers of non-negative second order elliptic operators, e.g., in the form $\mathcal{L}=-\nabla \cdot \mathbf{D} \nabla$, which are much more suited to the modelling of transport phenomena in complex domains.

Motivated by the promising results obtained in one spatial dimension (see [18]) and by the potential novel insight that fractional electrophysiological models could offer when used on realistic bounded geometries, in Section 3 we introduce a nonlocal modification of the Monodomain model that can take into account both the heterogeneity and the anisotropic character of the underlying spatial structure. We briefly describe the challenges linked to the use of nonlocal operators in bounded settings and show that the approach used in [16] can be naturally adapted to discretise the equations of interest considered here. We highlight the link between the integral representation of fractional powers of a non-negative second order elliptic operator and fractional powers of a suitable matrix deriving from a finite elements spatial discretisation of the problem, thus making a connection to the work in [17] (Section 4.1). As a result, we obtain a numerical tool that is flexible enough to handle both regular and possibly irregular realistic geometries, while being able to account for desirable properties in the modelling of electrical propagation in a complex structure. Some numerical results are shown in Section 5 and conclusions for this work are drawn in Section 6.

\section{Standard Monodomain model in electrocardiology}

Mathematical models of electrical signal propagation in cardiac electrophysiology consist of suitable spatially distributed formulations of specific ionic models reproducing the response of a single excitable cell to an applied electrical stimulus. Ionic models describe the temporal evolution of the transmembrane potential $u$ of a single excitable cell and the changes in $u$ caused by the opening and closing of various ion channels present in the cell membrane, driving the movement of ions into and out of the cell. A classical approach adopted in order to account for pulse propagation is to introduce spatial dependence via the Monodomain or the Bidomain model (e.g., see [1]). Both formulations consist of coupled PDE-ODE systems: while in the Monodomain case there is only one parabolic (typically nonlinear) PDE governing the transmembrane potential, the Bidomain involves a system of two PDEs (one nonlinear and parabolic, the other linear and elliptic) governing both the intracellular and the extracellular potentials.

A priori more accurate, the Bidomain is computationally more expensive due to its mathematical features: the matrix multiplying the time derivatives of the potentials is singular, inducing some difficulties in the numerical approximation of the system that lead to ill conditioned problems $[19,20]$. For these reasons many efforts have been devoted to set up efficient solvers and preconditioners [21, 22, 23, 24], possibly based on parallel multigrid approaches [25, 26, 27] or suitable approximations of the equations [28]. Potse and collaborators [29] compared the action potential propagation velocities using Bidomain and Monodomain and concluded that the latter propagates slightly slower (2\%). However, in absence of external fields, the Monodomain model is shown to be accurate enough to capture the desired dynamics and effects of the action potential propagation on the scale of a human heart (see $[29,30]$ ). Since the main difference in the propagation speed between the two models is localised at the propagation front but not along the fibre main directions, in recent years several studies have been devoted to efficiently couple the computationally cheaper Monodomain model with an accurate fibre description obtained from medical imaging $[31,32,33,34]$.

In light of the previous considerations, we base our study on the Monodomain model, and we briefly recall here its features. As the myocardial tissue is made of elongated cells surrounded 
by an extracellular medium and connected to each other via gap junctions forming a complex network of fibres, the local conductivity depends upon the fibres orientation, and in the most general case the associated tensor is anisotropic (see e.g. $[35,36]$ ). At any point $x \in \Omega$, where $\Omega$ is the spatial domain under consideration, it is thus possible to identify an orthonormal triplet of directions: parallel to the fibre direction, $\mathbf{a}_{l}(x)$, tangent, $\mathbf{a}_{t}(x)$, and orthogonal, $\mathbf{a}_{n}(x)$, to the radial lamination that is transversal with respect to the fibre axis.

Denoting by $\sigma_{l}, \sigma_{t}$, and $\sigma_{n}$ the conductivity coefficients in the $\mathbf{a}_{l}, \mathbf{a}_{t}$ and $\mathbf{a}_{n}$ directions, respectively, the conductivity tensor is given by

$$
\mathbf{D}(x)=\sigma_{l}(x) \mathbf{a}_{l}(x) \mathbf{a}_{l}^{T}(x)+\sigma_{t}(x) \mathbf{a}_{t}(x) \mathbf{a}_{t}^{T}(x)+\sigma_{n}(x) \mathbf{a}_{n}(x) \mathbf{a}_{n}^{T}(x) .
$$

It is usually assumed that $\mathbf{D}$ satisfies in $\Omega$ a uniform elliptic condition [1].

With these positions, the Monodomain model reads:

$$
\begin{array}{ll}
\chi C_{m} \frac{\partial u}{\partial t}-\nabla \cdot \mathbf{D} \nabla u+\chi I_{\text {ion }}(u, w)=I_{\text {stim }} & \text { in } \Omega \times(0, T) \\
\frac{d w}{d t}=R(u, w) & \text { in } \Omega \times(0, T) \\
u(x, 0)=u_{0}(x), \quad w(x, 0)=w_{0}(x) & \text { in } \Omega \\
\mathbf{n}^{T} \mathbf{D} \nabla u(x, t)=0 & \text { on } \partial \Omega \times(0, T),
\end{array}
$$

where $\chi$ is the cell surface-to-volume ratio, $C_{m}$ is the membrane capacitance per unit area, $I_{\text {ion }}$ is the sum of all transmembrane ionic currents, $I_{\text {stim }}$ is an applied stimulus, while $\mathbf{n}$ is the unit normal outward-pointing vector on the boundary surface $\partial \Omega$. In the above system, $u$ is the transmembrane potential, while $w$ is a vector collecting the state variables (such as gating and concentrations variables) of the ionic model under consideration, and whose dynamics we denote in general by $R(u, w)$. The homogeneous Neumann boundary condition on $\partial \Omega$ models an insulated myocardium. In this work, the numerical results of Section 5 were obtained for the Beeler-Reuter ionic model [37], but the methodology described in the following sections applies without modification to any ionic model.

\section{A Fractional Monodomain to Describe tissue heterogeneities}

In order to capture the effect of heterogeneities in the action potential propagation, we propose a space-fractional formulation of the Monodomain model that can be obtained by replacing the diffusion term in the parabolic equation with a nonlocal operator. Here we consider fractional powers of order $s \in(0,1)$, defined in the spectral sense, of the diffusion operator $\mathcal{L}=-\nabla \cdot \mathbf{D} \nabla$. The resulting nonlocal operators include the boundary conditions in their definition (homogeneous Neumann in the case at hand), and will be denoted by $\mathcal{L}_{\mathcal{N}}^{s}$, yielding

$$
\begin{array}{ll}
\chi C_{m} \frac{\partial u}{\partial t}+\mathcal{L}_{\mathcal{N}}^{s} u+\chi I_{\text {ion }}(u, w)=I_{\text {stim }} & \text { in } \Omega \times(0, T) \\
\frac{d w}{d t}=R(u, w) & \text { in } \Omega \times(0, T) \\
u(x, 0)=u_{0}(x), \quad w(x, 0)=w_{0}(x) & \text { in } \Omega .
\end{array}
$$

The crucial issue with fractional order differential operators is that they are naturally defined on the entire space $\mathbb{R}^{n}, n \geq 1$. However, in the majority of practical cases one needs to model quantities that are defined only on a bounded domain $\Omega \subset \mathbb{R}^{n}$. The main challenge is hence to 
suitably restrict, adapt, or interpret the definition of a fractional operator so that it preserves its nonlocal character while allowing for a well-posed formulation of the problem on $\Omega$. For this reason, we base our model on the spectral definition of fractional powers of $\mathcal{L}$.

3.1. Integral representation of $\mathcal{L}_{\mathcal{N}}^{s}$. Spectral fractional powers of $\mathcal{L}$ with exponent $s \in(0,1)$ are defined via the pairs $\left\{\lambda_{j}, \varphi_{j}\right\}_{j=0}^{\infty}$ of eigenvalues and orthonormal eigenfunctions of the operator $\mathcal{L}$ in $\Omega$, coupled to homogeneous Neumann boundary conditions on $\partial \Omega$. Specifically, if

$$
\left\{\begin{array}{l}
\mathcal{L} \varphi_{j}=\lambda_{j} \varphi_{j} \quad \text { in } \quad \Omega, \\
\mathbf{n}^{T} \mathbf{D} \nabla \varphi_{j}=0 \quad \text { on } \quad \partial \Omega,
\end{array}\right.
$$

with eigenfunctions such that $\left\|\varphi_{j}\right\|_{L^{2}(\Omega)}=1$, then the fractional power with exponent $s \in(0,1)$ of $\mathcal{L}$ coupled to the homogeneous Neumann boundary condition is defined as

$$
\mathcal{L}_{\mathcal{N}}^{s} v:=\sum_{j} \lambda_{j}^{s}\left\langle v, \varphi_{j}\right\rangle \varphi_{j}
$$

for all functions $v \in L^{2}(\Omega)$ such that $\sum_{j} \lambda_{j}^{2 s}\left|\left\langle v, \varphi_{j}\right\rangle\right|^{2}<\infty$, where $\langle\cdot, \cdot\rangle$ denotes the inner product in $L^{2}(\Omega)$.

On a bounded domain $\Omega$, rather than working with (5), one can use the heat-semigroup definition of the fractional operator (see [38]), stating that for all $x \in \Omega$,

$$
\mathcal{L}_{\mathcal{N}}^{s} v(x)=\frac{1}{\Gamma(-s)} \int_{0}^{\infty}(z(x, \tau)-v(x)) \frac{d \tau}{\tau^{1+s}},
$$

where $z(x, \tau)$ denotes the solution of the parabolic equation

$$
\begin{cases}\partial_{\tau} z+\mathcal{L} z=0 & (x, \tau) \in \Omega \times(0, \infty), \\ z(x, 0)=v(x) & x \in \Omega, \\ \mathbf{n}^{T} \mathbf{D} \nabla z=0 & (x, \tau) \in \partial \Omega \times[0, \infty) .\end{cases}
$$

We stress the fact that $\tau$ plays the role of time in the equation (7) but in reality it is simply the integration variable in the integral definition of $\mathcal{L}_{\mathcal{N}}^{s}$ and hence it must not be confused with the real temporal variable (denoted by $t$ ) in the parabolic problem (3).

Remark 1. Notice that, for a bounded domain $\Omega \subset \mathbb{R}^{n}$, expressions (5) and (6) remain valid in the more general case of $\mathcal{L}$ being a linear non-negative second-order partial differential operator, with discrete spectrum, densely defined and self-adjoint in $L^{2}(\Omega)[38]$.

\section{Numerical approximation of the Fractional Monodomain model}

We discretise in space the domain with a regular triangulation $\mathcal{T}_{h}$ and we consider a finite element space $V_{h}$ : let $\left\{\phi_{j}\right\}_{j=1}^{N_{h}}$ be a basis for $V_{h}$.

4.1. Spatial discretisation of the fractional operator. We focus here on the approximation of $\mathcal{L}_{\mathcal{N}}^{s} v$ for a given function $v$ on $\Omega$. We denote by $M \in \mathbb{R}^{N_{h} \times N_{h}}$ and $L \in \mathbb{R}^{N_{h} \times N_{h}}$ the symmetric mass and stiffness finite element matrices whose entries are given by

$$
M_{i j}=\sum_{K \in \mathcal{T}_{h}} \int_{K} \phi_{i} \phi_{j} \quad L_{i j}=\sum_{K \in \mathcal{T}_{h}} \int_{K}\left(\nabla \phi_{j}\right)^{T} \mathbf{D} \nabla \phi_{i} .
$$


The finite element solution of the parabolic equation (7) can be written as

$$
z_{h}(x, \tau)=\sum_{i=1}^{N_{h}} \mathbf{z}_{i}(\tau) \varphi_{i}(x),
$$

and the unknown vector $\mathbf{z}(\tau)=\left[\mathbf{z}_{1}(\tau), \ldots, \mathbf{z}_{N_{h}}(\tau)\right]^{T}$ satisfies the first order ODE system:

$$
\left\{\begin{array}{l}
M \mathbf{z}^{\prime}(\tau)=-L \mathbf{z}(\tau) \quad \tau \in(0, \infty), \\
\mathbf{z}(0)=\mathbf{v}
\end{array}\right.
$$

where $\mathbf{v}=\left[\mathbf{v}_{1}, \ldots, \mathbf{v}_{N_{h}}\right]^{T}$ collects the coefficients of the projection of $v$ onto the finite element space $V_{h}$.

Remark 2. The finite elements stiffness matrix defined in (8) naturally includes the homogeneous Neumann boundary condition. In the case different boundary conditions or more general elliptic operators were needed, the following argument would still hold, provided a suitable modification of $L$.

System (9) can be solved exactly leading to the following expression involving the initial condition of the system and a time-dependent matrix exponential:

$$
\mathbf{z}(\tau)=e^{-\tau A} \mathbf{v}
$$

with $A:=M^{-1} L$. We stress that, since $M$ is a real symmetric positive definite matrix, $A$ is well-defined. Moreover, since $L$ is also real symmetric and non-negative definite, it follows that $A$ has a real spectrum and is non-negative definite as well.

By substituting expression (10) in (6) we obtain the following discretisation in space of the fractional operator $\mathcal{L}_{\mathcal{N}}^{s} v$ :

$$
\begin{aligned}
\Theta^{s}[\mathbf{v}] & :=\frac{1}{\Gamma(-s)} \int_{0}^{\infty}\left(e^{-\tau A} \mathbf{v}-\mathbf{v}\right) \frac{d \tau}{\tau^{1+s}} \\
& =\left[\frac{1}{\Gamma(-s)} \int_{0}^{\infty}\left(e^{-\tau A}-\mathbb{I}_{N_{h}}\right) \frac{d \tau}{\tau^{1+s}}\right] \mathbf{v},
\end{aligned}
$$

where $\mathbb{I}_{N_{h}} \in \mathbb{R}^{N_{h} \times N_{h}}$ denotes the identity matrix and the second equality follows from the vector $\mathbf{v}$ being independent from the integration variable $\tau$.

Lemma 1. If a matrix $B \in \mathbb{R}^{N \times N}$ is non-negative definite and diagonalisable, then

$$
B^{s}=\frac{1}{\Gamma(-s)} \int_{0}^{\infty}\left(e^{-\tau B}-\mathbb{I}_{N}\right) \frac{d \tau}{\tau^{1+s}} .
$$

Proof. Let $B=V \Lambda V^{-1}$ be a diagonalisation of $B$ where $\Lambda=\operatorname{diag}\left(\lambda_{1}, \ldots, \lambda_{N}\right)$ is the diagonal matrix of the eigenvalues of $B$, and $V$ is the matrix whose $j$-th column is the eigenvector corresponding to $\lambda_{j}$ for $j=1, \ldots, N$. If $f$ is a function defined on the spectrum of $B$, then $f(B)$ can be written as

$$
f(B)=V f(\Lambda) V^{-1},
$$

where $f(\Lambda):=\operatorname{diag}\left(f\left(\lambda_{1}\right), \ldots, f\left(\lambda_{N}\right)\right)$. Moreover, for all $\lambda \geq 0$, by integration by parts and by using the definition of the function $\Gamma$, it can be shown that

$$
\lambda^{s}=\frac{1}{\Gamma(-s)} \int_{0}^{\infty}\left(e^{-\tau \lambda}-1\right) \frac{d \tau}{\tau^{1+s}} .
$$


Therefore, combining (14) and (15) it is straightforward to see that the matrix function within square brackets in (12) is exactly the fractional power, with exponent $s$, of the matrix $B$.

Owing to the previous Lemma, the space discrete counterpart of $\mathcal{L}_{\mathcal{N}}^{s} v$ for a given function $v$, previously denoted by $\Theta^{s}[\mathbf{v}]$, is nothing but the matrix-vector product $A^{s} \mathbf{v}$.

4.2. Semi-discrete Fractional Monodomain. The semi-discrete unknowns of system (3) on the grid nodes are given by

$$
\mathbf{u}(t)=\left[\begin{array}{c}
\mathbf{u}_{1}(t) \\
\vdots \\
\mathbf{u}_{N_{h}}(t)
\end{array}\right] \quad \mathbf{w}_{j}(t)=\left[\begin{array}{c}
\mathbf{w}_{j, 1}(t) \\
\vdots \\
\mathbf{w}_{j, N_{h}}(t)
\end{array}\right] j=1, \ldots, p,
$$

where $p$ denotes the number of state variables in $w$.

The semi-discrete variables are then given by

$$
u_{h}(x, t)=\sum_{i=1}^{N_{h}} \mathbf{u}_{i}(t) \varphi_{i}(x) \quad w_{j, h}(x, t)=\sum_{i=1}^{N_{h}} \mathbf{w}_{j, i}(t) \varphi_{i}(x) \quad j=1, \ldots, p .
$$

By letting $\mathbf{w}=\left[\mathbf{w}_{1}^{T}(t), \ldots, \mathbf{w}_{p}^{T}(t)\right]^{T}$, the spatial discretisation of $(3)$ can be rewritten as:

$$
\begin{array}{ll}
\chi C_{m} \frac{d \mathbf{u}}{d t}+A^{s} \mathbf{u}+\chi I_{\text {ion }}(\mathbf{u}, \mathbf{w})=I_{\text {stim }} & \text { in }(0, T) \\
\frac{d \mathbf{w}}{d t}=R(\mathbf{u}, \mathbf{w}) & \text { in }(0, T)
\end{array}
$$

coupled to the initial conditions $\mathbf{u}(0)=\mathbf{u}_{0}$ and $\mathbf{w}(0)=\mathbf{w}_{0}$.

The advantage of considering the expression in (12) rather than just looking at (11) is that this formulation will allow us to use a semi-implicit temporal scheme for the numerical solution of the Monodomain equation (the diffusive term is considered at the current time step and only the reaction part is evaluated at the previous time iteration), thus avoiding the severe time step restriction that a fully explicit scheme would require for stability.

4.3. Time marching scheme. The ODE system (16) is discretised in time via a semi-implicit Euler scheme in which the diffusion term is treated implicitly while the reaction term is evaluated at the current time step. Let $\Delta t>0$ and $N_{t}=T / \Delta t$ : for the sake of simplicity in presentation, we consider here a uniform grid of time points $t_{n}=n \Delta t$ with $n=0, \ldots, N_{t}$, but adaptive schemes in time can be considered as well, and will be the object of further investigation. We denote $\mathbf{u}^{(n)}:=\mathbf{u}\left(t_{n}\right)$ and $\mathbf{w}^{(n)}:=\mathbf{w}\left(t_{n}\right)$. The value of $\mathbf{u}^{(n+1)}$ and $\mathbf{w}^{(n+1)}$ is computed at each time step in two stages following the strategy proposed by Whiteley [39]. First, we compute the updated value of the transmembrane potential by solving the first equation in (16), involving the fractional operator $A^{s}$ :

$$
\chi C_{m} \frac{\mathbf{u}^{(n+1)}-\mathbf{u}^{(n)}}{\Delta t}+A^{s} \mathbf{u}^{(n+1)}+\chi I_{i o n}\left(\mathbf{u}^{(n)}, \mathbf{w}^{(n)}\right)=I_{\text {stim }} .
$$

Then, we use the newly computed $\mathbf{u}^{(n+1)}$ in the second equation in (16). This way, most of the ODEs involved (corresponding to the six gating variables for the Beeler-Reuter ionic model) become linear in the corresponding differentiation variable. Hence, although an implicit scheme is used, the updated value of $\mathbf{w}^{(n+1)}$ can be explicitly expressed in terms of only $\mathbf{u}^{(n+1)}$ and $\mathbf{w}^{(n)}$. Only a small nonlinear system corresponding to the remaining variables (the calcium concentration for the Beeler-Reuter model) needs to be solved at each time step: in our numerical simulations we used the modified Newton method with the Jacobian evaluated at the first iteration. 
By rewriting equation (17) as

$$
\left(\mathbb{I}_{N_{h}}+\frac{\Delta t}{\chi C_{m}} A^{s}\right) \mathbf{u}^{(n+1)}=\mathbf{b}^{(n)},
$$

with

$$
\mathbf{b}^{(n)}:=\mathbf{u}^{(n)}+\frac{\Delta t}{C_{m}}\left[-I_{i o n}\left(\mathbf{u}^{(n)}, \mathbf{w}^{(n)}\right)+\frac{1}{\chi} I_{\text {stim }}\right],
$$

it is immediate to see that at each time step the approximation of $f(A) \mathbf{b}^{(n)}$ with

$$
f(A):=\left(\mathbb{I}_{N_{h}}+\frac{\Delta t}{\chi C_{m}} A^{s}\right)^{-1}
$$

is needed.

4.4. Contour integral method. The solution of equation (18) is computed with one of the approaches proposed in [17], namely the Contour Integral (CI) method. This method is based on the expression of $f(A)$ as a contour integral in the complex plane winding around the spectrum of $A$ in a counterclockwise direction.

This integral is approximated via a suitable quadrature formula with $n_{\mathrm{CIM}}$ nodes so that, given a matrix $A \in \mathbb{R}^{N \times N}$ and a vector $b \in \mathbb{R}^{N}$, one has

$$
f(A) \mathbf{b} \approx f_{n_{\mathrm{CIM}}}(A) \mathbf{b}=\sum_{r=1}^{n_{\mathrm{CIM}}} \omega_{r}\left(\xi_{r} \mathbb{I}_{N}-A\right)^{-1} \mathbf{b},
$$

where $\omega_{r}$ and $\xi_{r}$ represent suitable quadrature weights and complex shifts, respectively.

A detailed discussion on the implementation of the CI method is out of the scope of the present paper and we refer the reader to [40] and in particular to [17] for the case of interest, i.e., the case of a singular matrix $A$ due to the homogeneous Neumann boundary conditions imposed. Nevertheless, we recall some important features of the method.

The computation of $f_{n_{\text {CIM }}}(A) \mathbf{b}$ requires the solution of $n_{\text {CIM }}$ independent linear systems of the form $\left(\xi_{r} \mathbb{I}_{N}-A\right) \mathbf{y}=\mathbf{b}$. Since $A=M^{-1} L$, the considered linear systems can be equivalently rewritten as $\left(\xi_{r} M-L\right) \mathbf{y}=M \mathbf{b}$, which in turn can be efficiently solved exploiting the fact that $M$ and $L$ are sparse and easily assembled finite element matrices. Hence, the full matrix $A$ is never formed. In addition, the solution of the $n_{\mathrm{CIM}}$ linear systems can be implemented in parallel.

Let $f$ be an analytic function in $\mathbb{C} \backslash(-\infty, 0]$. When $A$ is a real positive definite matrix, the number of quadrature nodes of the CI method needed to obtain a specified accuracy in the approximation of $f(A) \mathbf{b}$ increases asymptotically as $\log \left(\lambda_{1} / \lambda_{N}\right)$ (see Section 3.1 of Burrage et al. [17]), with $\lambda_{1}$ and $\lambda_{N}$ denoting the minimum and maximum eigenvalues of $A$, respectively. In fact, as shown by Hale and colleagues (Theorem 2.1 in [40], here rewritten according to our notation),

$$
\left\|f(A)-f_{n_{\text {CIM }}}(A)\right\|=O\left(e^{-\pi^{2} n_{\text {CIM }} /\left(\log \left(\lambda_{N} / \lambda_{1}\right)+3\right)}\right) .
$$

When homogeneous Neumann boundary conditions are considered (the real matrix $A$ is only semi-positive definite and $\lambda_{1}=0$ ), similar considerations can be made. This time, to obtain a specified accuracy $n_{\mathrm{CIM}}$ increases asymptotically as $\log \left(\lambda_{2} / \lambda_{N}\right)$, with $\lambda_{2}$ being the first positive eigenvalue of $A$ (see relative discussion in Section 4 of Burrage et al. [17]).

Note that once again, when $A=M^{-1} L$ with sparse $M$ and $L$, the eigenvalues $\lambda_{2}$ and $\lambda_{N}$ can be efficiently estimated without the need of assembling the matrix $A$ (e.g., see Chapter 5 in [41]).

Remark 3. In the classical Monodomain the update of the potential only requires the solution of one real linear system at each time step. The computational cost required by the solution of the fractional Monodomain model instead can be quantified by the initialisation step (to identify the 
quadrature weights $\omega_{r}$ and the shifts $\left.\xi_{r}\right)$ and the $n_{\mathrm{CIM}}$ complex linear systems that have to be solved at each time iteration.

\section{Numerical Results}

In this section we present some numerical simulations to illustrate the features of the fractional Monodomain. The nonlocality of the fractional operator requires a finer mesh, compared to the classical differential operator, in order to capture sharp propagation fronts, typical of electrophysiology. This drawback was already evident for one-dimensional problems (see, e.g. [18]). Resorting to a finer mesh is an adequate solution, at the cost of higher CPU times. We present here numerical simulations on two dimensional domains, discretised with unstructured meshes. Our numerical results show that a two-dimensional setting is already sufficient to appreciate the effect of using a fractional operator in the propagation of electrophysiological waves. We describe the myocytes behaviour by the Beeler-Reuter model [37], and we consider a time step $\Delta t=0.1 \mathrm{~ms}$, which is sufficiently accurate to capture its action potential dynamics. We trigger a localized stimulus of 2 $\mathrm{ms}$ at the beginning of the test, and we run the simulation until the whole domain has recovered to its initial resting condition.

We run simulations for different values of the fractional parameter $s$ : we choose $s=1$ (classical Monodomain), and $s=0.9,0.8,0.7$ (fractional cases). Although smaller values could be considered, we observe that these choices of $s$ are sufficient to obtain a meaningful comparison between the local and the nonlocal cases, and to highlight significant trends in the results.

We consider four different test cases. In the first three tests, the computational domain is $\Omega=[0,1] \times[0,1] \mathrm{cm}^{2}$, discretised by an unstructured grid consisting of 69177 nodes and 137552 elements, with a characteristic mesh size $h_{\max }=0.0076 \mathrm{~cm}$. In order to minimise the effect of the boundary in the APD dispersion, we consider the initial stimulus to occur in the bottom left corner of $\Omega$, namely in a circle of radius $r=0.1 \mathrm{~cm}$ centred in $(0,0)$. We highlight the effects of the fractional operator in terms of the difference in propagation speed and the APD dispersion. The first test considers an isotropic domain, while the second and third tests account for fibres directionality. Finally, the fourth test is performed on a cardiac slice.

All our numerical simulations were performed in MATLAB (R2016a, The MathWorks Inc., Natick, Massachusetts, US). In particular, the CI method was implemented following the lines of code provided in [17] and using the subroutines from Driscoll's Schwarz-Christoffel toolbox [42] for the Jacobi elliptic functions required in the computation.

5.1. Computational considerations on the Contour Integral method. We devote this section to discuss the computational accuracy and efficiency of the CI method. Let $A=M^{-1} L$, where $M$ and $L$ denote respectively the finite element mass and stiffness matrices of the problem associated with the computational domain $\Omega=[0,1] \times[0,1] \mathrm{cm}^{2}$, that is used in the numerical tests presented in the following Sections 5.2-5.4. Let $f(A)$ be as in equation (20), where $\Delta t=0.1$ ms, while the other coefficients are given in Table 1 . We test the performance of the CI method in the approximation of $f(A) \mathbf{b}$ with $s=0.9,0.8,0.7$, for different choices of the vector $\mathbf{b}$, different matrices $A$, and different values of the parameter $n_{\mathrm{CIM}}$.

In studying the accuracy of the method, we first consider the case of an isotropic tissue with constant conductivity $\sigma$ (as described in detail in Test 1 - Section 5.2). We consider here $\mathbf{b}$ as the right-hand side of equation (19) with $\mathbf{u}^{(n)}$ and $\mathbf{w}^{(n)}$ representing the numerical solution of the classical Monodomain model computed at three different time points. Specifically, we choose the time points $t=100, t=200$, and $t=300 \mathrm{~ms}$ in order for the vector $\mathbf{b}$ to reflect different moments in the spatial dynamics of the membrane potential. We study the computational error in function of the number of quadrature points $n_{\mathrm{CIM}}$. Following Burrage et al. [17], the exact value of $f(A) \mathbf{b}$ 
is replaced by a reference solution computed with $n_{\mathrm{CIM}}=100$ and denoted by $f_{100}(A) \mathbf{b}$. Figure 1 shows the relative error

$$
\frac{\left\|f_{100}(A) \mathbf{b}-f_{n_{\mathrm{CIM}}}(A) \mathbf{b}\right\|_{\infty}}{\left\|f_{100}(A) \mathbf{b}\right\|_{\infty}}
$$

as a function of $n_{\mathrm{CIM}}$ for the different vectors $\mathbf{b}$ considered. In each plot, solid lines represent the relative error while the dashed line is the exponentially decaying function $e^{-\pi^{2} n_{\mathrm{CIM}} /\left(\log \left(\lambda_{N} / \lambda_{2}\right)+3\right)}$ (which does not depend on the particular choice of $s$ nor $\mathbf{b}$ ).
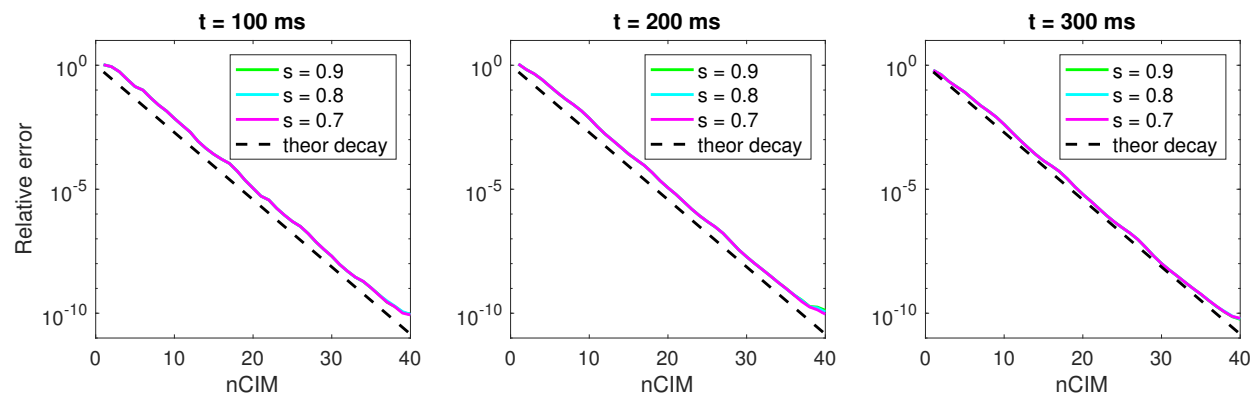

Figure 1. Relative error decay in the computation of $f(A) \mathbf{b}$ as a function of the number of quadrature points in the CI method. $A$ is fixed, $f$ as in equation (20), $\mathbf{b}$ computed as the right-hand side of the classical Monodomain equation at $t=100$ $\mathrm{ms}$ (left), $t=200 \mathrm{~ms}$ (centre), and $t=300 \mathrm{~ms}$ (right).

For each of the three cases, the relative errors in the computation of $f(A) \mathbf{b}$ for different values of the fractional parameter $s$ are practically indistinguishable, indicating that the performance of the method is independent from the values of $s$ considered. Moreover, in all cases the numerical error matches very well the predicted theoretical decay showing a consistent performance also as the simulation time advances.

We then study the performance of the CI method under different tissue characteristics. In particular, we consider the case of anisotropic tissue with fibres aligned with the $x$ axis (as in Test 2 - Section 5.3) and anisotropic tissue with rotating fibres (as in Test 3 - Section 5.4). Different assumptions on the isotropic character of the tissue correspond to modifications of the stiffness matrix $L$. Hence, the eigenvalues $\lambda_{2}$ and $\lambda_{N}$ of the matrix $A$ change accordingly, in general yielding different slopes in the exponential decay of the error (22). In Figure 2 we show the results for $s=0.8$ and the vector $\mathbf{b}$ being the right-hand side of equation (19) obtained via the classical Monodomain at $t=200 \mathrm{~ms}$ (similar results were obtained for $s=0.9,0.7$ and $t=100,300 \mathrm{~ms}$ ). In all cases, the expected theoretical behaviour is well matched by the numerical experiments.

We now turn our attention to the computational cost of the CI method given by the initialisation phase (identification of the quadrature weights $\omega_{r}$ and the shifts $\xi_{r}$ ), and by the $n_{\mathrm{CIM}}$ linear systems that need to be solved at each time step. Since these systems are independent from each other, they are well-suited for a parallel implementation of their solution. Although needed in the case of large three-dimensional problems, massive parallelisation is beyond the scope of this paper, but some considerations are still in order. Given the reasonable size of the linear system to be solved, we consider here only the scenario in which each system is assigned to one core, thus minimising the communication between processors. Figure 3 shows the cost of computing the matrix function vector product $f_{n_{\text {CIM }}}(A) \mathbf{b}$ for $s=0.9,0.8,0.7$, as a function of $n_{\mathrm{CIM}}$, when 1,5 , or 10 cores of the same multi-core machine are used in the computation. The vector $\mathbf{b}$ is the right-hand side of equation (19) obtained via the classical Monodomain at $t=100 \mathrm{~ms}$ (once again analogous results 

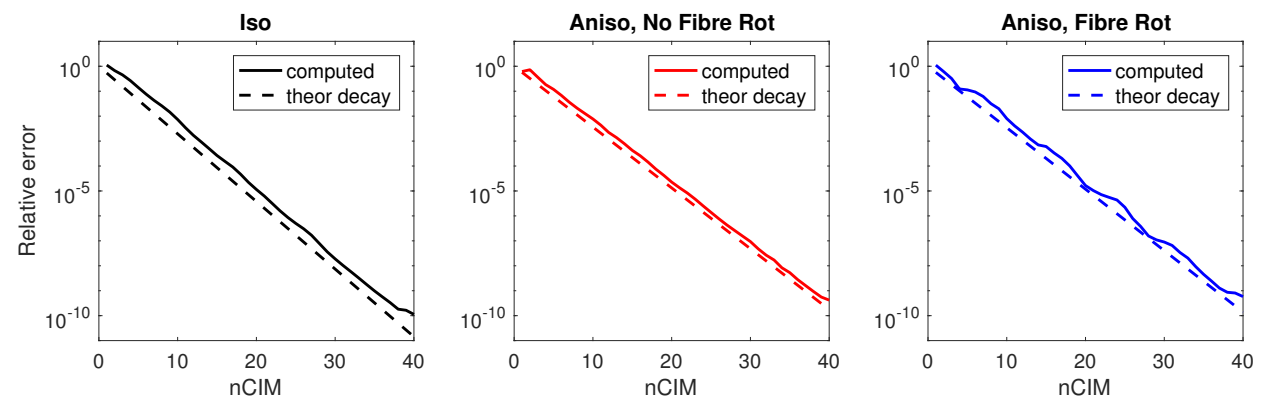

FiguRE 2. Relative error decay as a function of the number of quadrature points in the CI method under different tissue assumptions. Left: Isotropic case (Test 1). Centre: Anisotropic with fibres aligned to the $x$ axis (Test 2). Right: Anisotropic with rotating fibres (Test 3 ).
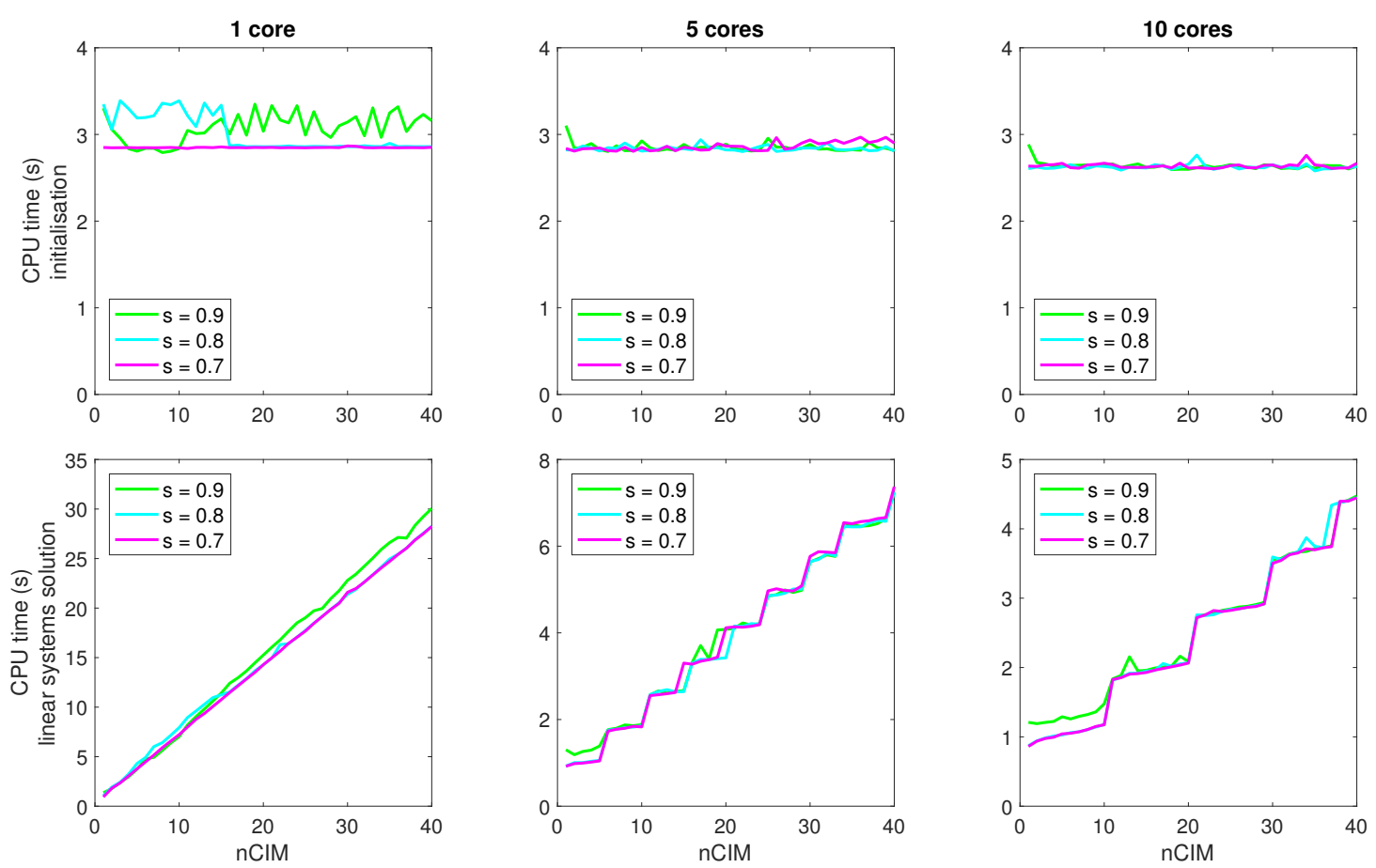

Figure 3. CPU times for the computation of $f_{n_{\mathrm{CIM}}}(A) \mathbf{b}$ with $s=0.9,0.8,0.7$ as a function of $n_{\text {CIM }}$ on 1 core (left), 5 cores (centre), and 10 cores (right), respectively. Top row: CPU time required for the initialisation of the CI method. Bottom row: CPU time of the actual solution of the $n_{\mathrm{CIM}}$ independent linear systems.

were obtained for $t=200,300 \mathrm{~ms}$ ). Since the initialisation phase of the CI method depends on the value of $n_{\text {CIM }}$ and hence had to be performed before each computation, we split its cost from the cost of solving the linear systems in order to obtain a clearer picture.

We observe that CPU performance is essentially independent of the particular value of $s$ considered. Moreover, while there is a clear reduction of CPU times when multiple cores are available, 
the initialisation cost is not significantly reduced by parallelisation and could hence take its toll in terms of the total cost required by the CI method. However, owing to Figure 1 and Figure 2, the same number of quadrature points $n_{\mathrm{CIM}}$ can be used for the entire simulation of spatial propagation via the fractional Monodomain, so that the CI initialisation has to be performed only once. Consequently, the efficiency of the algorithm is solely determined by the amount of resources available for parallelisation. In particular, as the $n_{\text {CIM }}$ linear systems are expected to be equivalent in terms of computational cost, it is reasonable to assume that the ideal solution is to use a number of cores equal to $n_{\mathrm{CIM}}$ in order to evenly distribute the computational load among cores, as well as minimise communication and memory requirements.

In light of the above considerations, we set $n_{\mathrm{CIM}}=24$ in all our simulations, as it appears to generate a sufficiently accurate approximation (relative error of the order of $10^{-5}$ ) in all considered scenarios, and we implement the CI method on 24 cores of the BCAM cluster by using the Parallel Computing Toolbox of MATLAB.

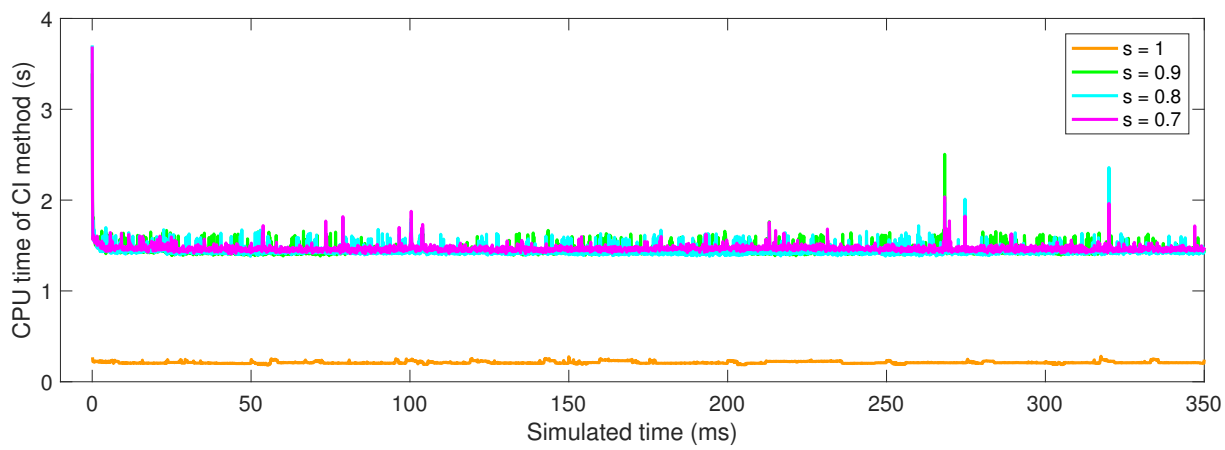

FIgure 4. Computational cost at each time step of the solution of the classical Monodomain equation $(s=1)$ and of the fractional Monodomain (with $s=0.9,0.8,0.7)$ as the simulation time advances. The fractional case is solved by setting $n_{\mathrm{CIM}}=24$ and parallelising the solution of the 24 linear systems on 24 cores.

Finally, in Figure 4 we show the computational cost required at each time step by the solution of the single linear system in the classical Monodomain and by the solution of the fractional Monodomain (with $s=0.9,0.8,0.7$ ), for the first $350 \mathrm{~ms}$ of simulated time. The settings of the problem are the ones of Test 1 in the forthcoming Section 5.2. The extra computational cost of the fractional Monodomain compared to the classical Monodomain is clearly visible, but the performance of the CI algorithm is practically independent from the value of the fractional parameter $s$. The weight of the initialisation phase of the CI method is evident in the first step of the simulation. However, it plays no role in the rest of the simulation where the computational cost is determined by the parallelised solution of the 24 linear systems. In all cases, the performance of the algorithm remains fairly constant during the whole simulation.

5.2. Test 1: isotropic tissue. In the first test, we consider an isotropic tissue. Notice that in this case we have $\mathcal{L}=-\sigma \Delta$, for some constant conductivity $\sigma>0$.

The smaller the value of $s$ the stronger is the deviation from homogeneous tissue $(s=1)$ : as a consequence, we expect both the propagation speed to reduce and the APD dispersion to increase with the reduction of $s$. To assess the difference in propagation speed of the excitation wave, we set $\sigma=0.1 \mathrm{mS} \cdot \mathrm{cm}^{-1}$, we randomly select 100 nodes in $\Omega$ (see Figure 5 , left), and consider their 
activation time, as a function of distance from the stimulus region, for different values of $s$. In Figure 5 (top right) we can see the expected behaviour of the activation time, indicating that the propagation gets slower as $s$ decreases. In the same figure (bottom right) we plot the action potential in the centre of the domain $(0.5,0.5)$ for the considered values of $s$ : once again, we notice an increasing delay in the activation of the node, as $s$ is reduced. Moreover, in line with the results for the one-dimensional case [18] we observe a reduction in both spike height and AP duration as $s$ decreases, as well as an increase in width of the AP foot. This was also observed in [5], where a fractional model with order $\alpha=1.75(s=\alpha / 2=0.875$ according to our notation) was shown to best replicate the effects of wavefront conduction in tissue depolarization of observed experimental data, and in particular AP foot morphology.
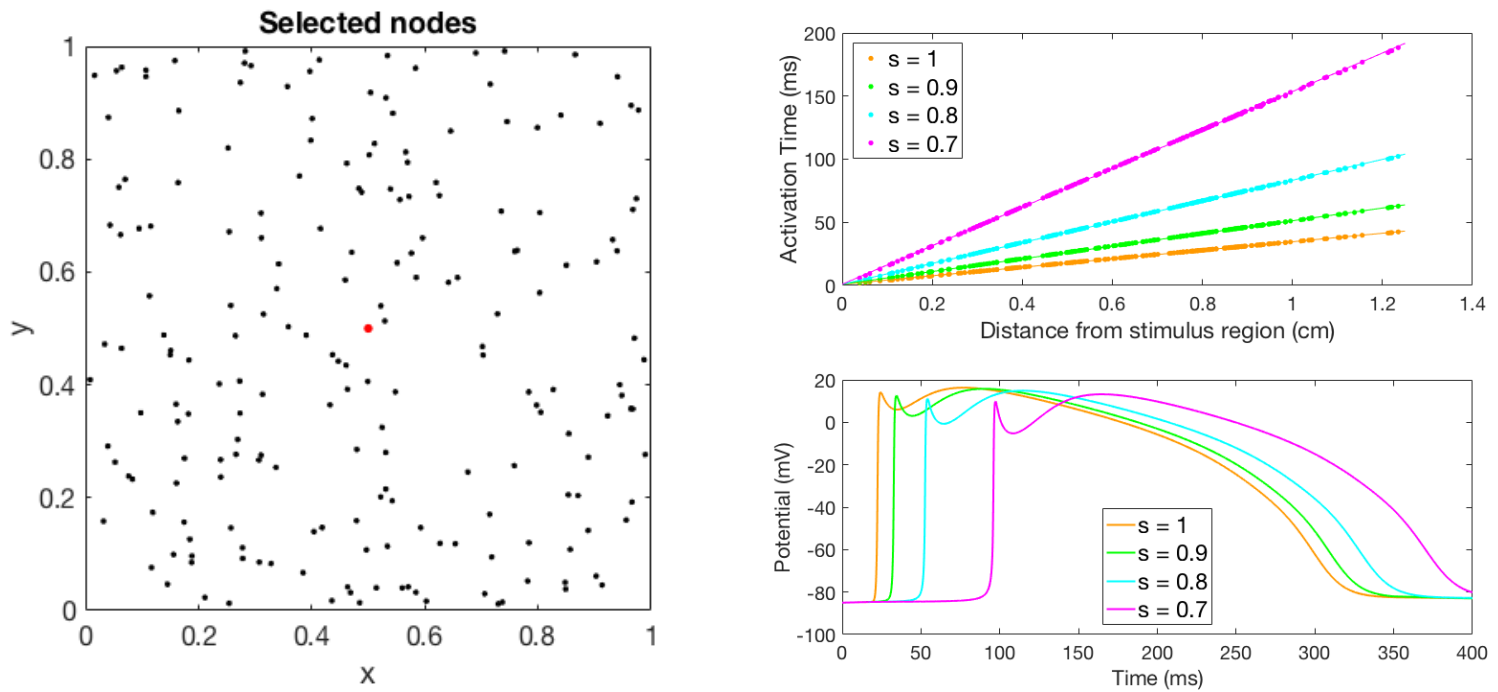

Figure 5. Isotropic tissue. Activation time and action potential. Left: location of the 100 randomly selected nodes. Right: activation times (top) in the random points for different values of $s$, and action potentials (bottom) in point $(0.5,0.5)$, highlighted in red.

As stated in [5], "at the tissue level, macroscopic properties such as conduction velocity must be captured by the specific propagation model, regardless of its mathematical description". In order to obtain comparable conduction velocities and hence assess the APD dispersion on comparable activation times for all values of $s$, in the second part of this test we rescale the conductivity coefficient $\sigma$ of the Fractional Monodomain with non-integer $s$ (see Table 1) so that full domain activation is obtained at the same time of the case $s=1$.

In Figure 6 (top left) we plot the APD dispersion (in ms) as the excitation wave propagates away from the source. All cases exhibit dispersion but, while in the classical $s=1$ case this occurs only near the stimulus site and when the wave approaches the far boundary, in the remaining cases this feature is evident in the whole $\Omega$, and is more pronounced as $s$ decreases. In the remaining plots of Figure 6 we show the APD dispersion along three different lines: the main diagonal $y=x$, $y=0.5 x$, and the lower border $y=0$. The more pronounced effect is observable along the diagonal, which is not surprising, due to the longer path the wave has to travel before experiencing boundary effects. 

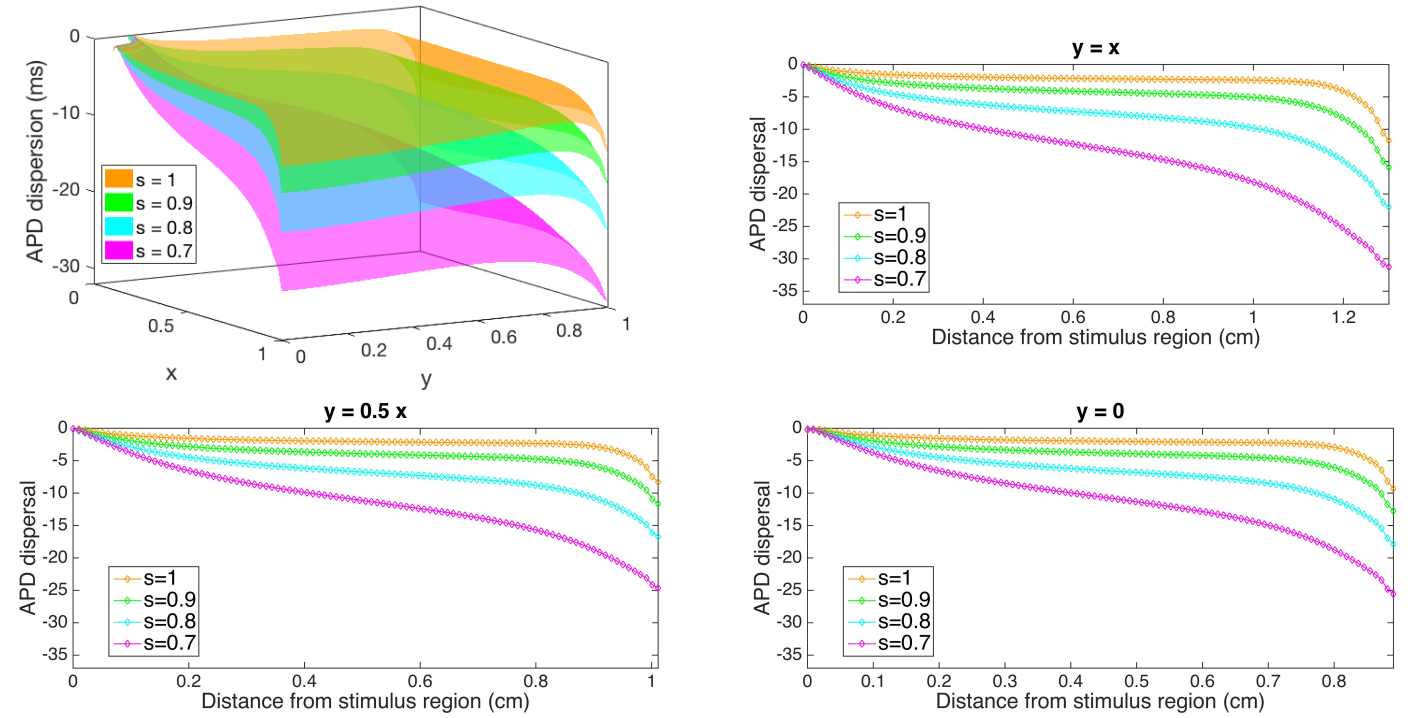

Figure 6. Isotropic tissue. APD dispersion. Top left: APD dispersion on the computational domain $\Omega$ for different values of $s$. Top right to bottom right: APD dispersion along three different lines $(y=x, y=0.5 x$, and $y=0)$.

Once again this is consistent with previous numerical results obtained in the one-dimensional case in [18] and with the shortening of APD during propagation (known as inverse AT-APD relationship) present in experimental data and well matched in [5] by a one-dimensional fractional diffusion model.

\begin{tabular}{|c|c|c|c|c|c|c|}
\hline General & \multicolumn{6}{|c|}{$C_{m}=1 \mu \mathrm{F} \cdot \mathrm{cm}^{-2} \quad \chi=2000 \mathrm{~cm}^{-1}$} \\
\hline Stimulus & \multicolumn{6}{|c|}{$I_{\mathrm{stim}}=10^{5} \mu \mathrm{A} \cdot \mathrm{cm}^{-3}$} \\
\hline Source & \multicolumn{3}{|c|}{$x^{2}+y^{2}<r^{2}$} & \multicolumn{3}{|l|}{$r=0.1$} \\
\hline \multirow{4}{*}{ Conductivities } & $s$ & 1 & 0.9 & 0.8 & 0.7 & Units \\
\hline & $\sigma$ & 0.1 & 0.2354 & 0.6246 & 2.1396 & $\mathrm{mS} \cdot \mathrm{cm}^{-1}$ \\
\hline & $\sigma_{l}$ & 0.2 & 0.4620 & 1.2780 & 4.5550 & $\mathrm{mS} \cdot \mathrm{cm}^{-1}$ \\
\hline & $\sigma_{t}$ & 0.02 & 0.0462 & 0.1278 & 0.4555 & $\mathrm{mS} \cdot \mathrm{cm}^{-1}$ \\
\hline
\end{tabular}

TABLE 1. Model parameters for Tests 1-3.

5.3. Test 2: anisotropic tissue. In this second test we consider anisotropic tissue with fibres aligned with the $x$ axis, namely $\mathbf{a}_{l}(x, y)=[1,0]^{T}$ and $\mathbf{a}_{t}(x, y)=[0,1]^{T}$. In this case the fractional operator is the $s$-th power of $\mathcal{L}=-\nabla \cdot \mathbf{D} \nabla$. We assume constant conductivities both along $\left(\sigma_{l}\right)$ and across $\left(\sigma_{t}\right)$ the fibres, with $\sigma_{l}=10 \sigma_{t}$. As we did in the second part of the previous test, we rescale the coefficients (see Table 1) so that the activation times are comparable (Figure 7, top left) and we analyse the APD dispersion induced by the fractional operator for different values of $s$. As in the isotropic case, we observe APD dispersion in the whole domain $\Omega$ for $s<1$, while the feature is evident only near the stimulus and near the boundary in the classical case $s=1$. Moreover, it can be observed (Figure 7, top right to bottom right) that the APD dispersion gets 
more pronounced as one moves away from the principal direction of fibres (line B vs line C), and the largest dispersion occurs in the direction normal to the fibres (line A).

5.4. Test 3: anisotropic tissue with rotating fibres. In this third test we consider again anisotropic conductivity $\left(\sigma_{l}=10 \sigma_{t}\right)$, but with fibres continuously rotating counterclockwise around the point $(0,1)$, see Figure 8 (top left). Namely, we consider for $(x, y) \in \Omega$ :

$$
\mathbf{a}_{l}(x, y)=\left[\cos \frac{\pi x}{2}, \sin \frac{\pi x}{2}\right]^{T}, \quad \mathbf{a}_{t}(x, y)=\left[-\sin \frac{\pi x}{2}, \cos \frac{\pi x}{2}\right]^{T} .
$$

Once again, the coefficients are rescaled as in Test 2 so that the activation times are comparable and we analyse the APD dispersion induced by the fractional operator. In Figure 8 (top right) we plot the activation time, highlighting how the propagation speed reflects the stronger conductivity along the principal fibre direction. In Figure 8 (bottom) we plot the APD dispersion along curve A (left), a vertical line next to the left boundary of $\Omega$, and along curve B (right) that follows the principal fibre direction. Again, it can be observed that the APD dispersion is less pronounced along the principal fibre direction. This feature can be globally observed in Figure 9, where we plot the APD dispersion on the whole $\Omega$ for the considered values of $s$.

5.5. Test 4: cardiac slice. In this last test we use as computational domain $\Omega$ a geometry obtained via manual segmentation of a real cardiac slice image taken from [43]. The domain $\Omega$ is discretized by an unstructured triangular grid consisting of 375489 nodes and 745579 elements, with a characteristic mesh size $h_{\max }=0.01 \mathrm{~cm}$. Not having a fibres description available for the particular geometry considered, we assume in this test an isotropic conductivity tensor $\mathbf{D}=\sigma \mathbb{I}_{2}$. If fibre directions were available, they would be easily incorporated by using the technique of the
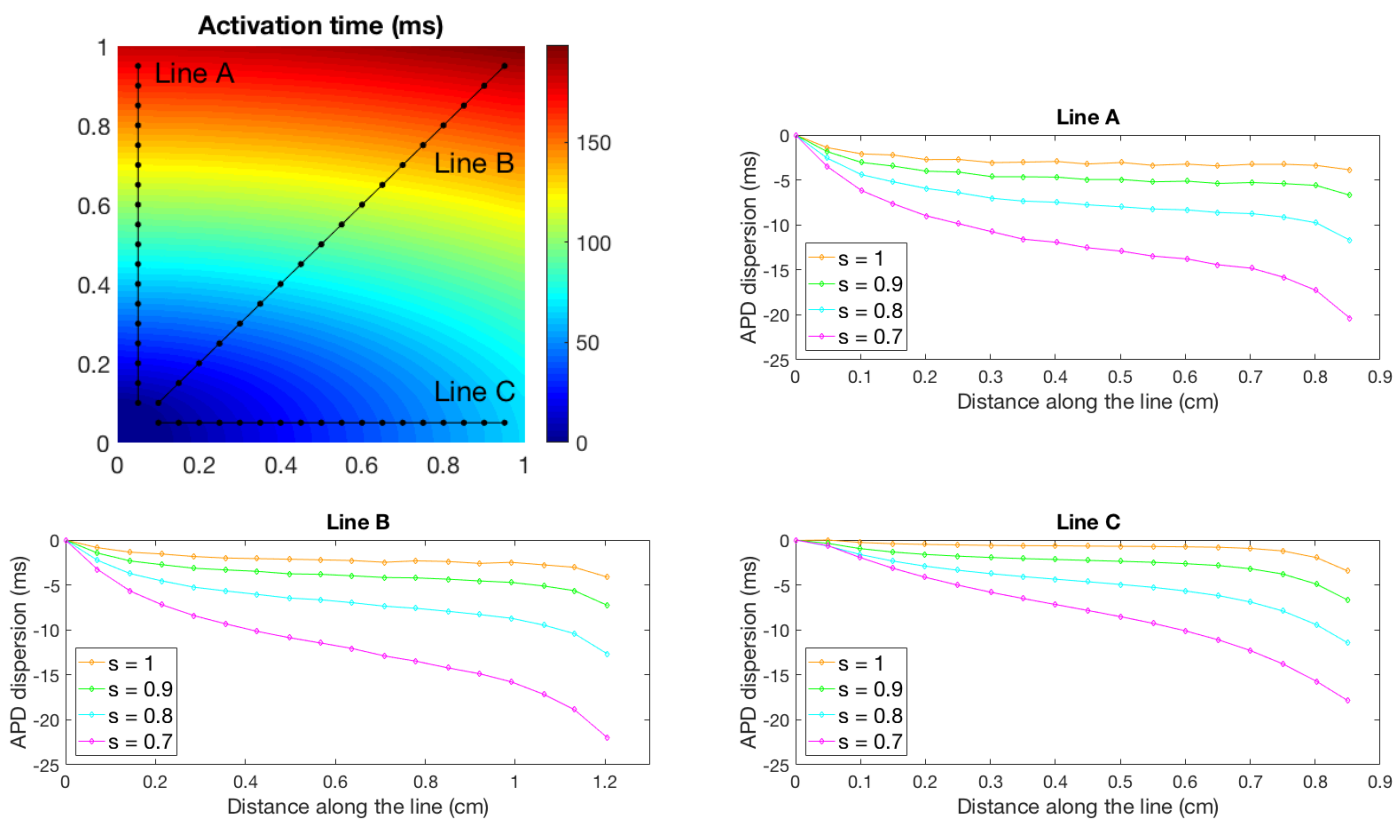

Figure 7. Test 2. Anisotropic tissue: activation time and APD dispersion. Top left: activation time. Top right to bottom right: APD dispersion along lines A, $\mathrm{B}$, and $\mathrm{C}$. 
previous test cases. Once again $\sigma$ has been rescaled among the considered values of $s$, in order to have comparable activation times, that we plot in Figure 10. We refer to Table 2 for the parameters used in this case.

In Figure 11 we plot the APD dispersion on the slice for the considered values of $s$. All cases exhibit dispersion around the source, and close to the far boundaries. Dispersion is also particularly evident in regions where two wavefronts collide. This feature could not be observed in the previous tests, since our settings did not trigger any colliding waves. Once again, for $s<1$ APD dispersion is observed in the whole domain $\Omega$, and is increasing as $s$ gets smaller.

\begin{tabular}{|l|c|c|c|c|c|c|}
\hline General & \multicolumn{4}{|c|}{$C_{m}=1 \mu \mathrm{F} \cdot \mathrm{cm}^{-2}$} & $\chi=2000 \mathrm{~cm}^{-1}$ \\
\hline Stimulus & \multicolumn{5}{|c|}{$I_{\text {stim }}=10^{5} \mu \mathrm{A} \cdot \mathrm{cm}^{-3}$} & \\
\hline Source & \multicolumn{5}{|c|}{$(x-3.25)^{2}+(y-0.5)^{2}<r^{2}$} & $r=0.05$ \\
\hline \multirow{2}{*}{ Conductivities } & $s$ & 1 & 0.9 & 0.8 & 0.7 & Units \\
\cline { 2 - 7 } & $\sigma$ & 1 & 2.354 & 6.246 & 21.396 & $\mathrm{mS} \cdot \mathrm{cm}^{-1}$ \\
\hline
\end{tabular}

TABLE 2. Model parameters for Test 4.

\section{Conclusions}

In this paper we introduce a space-fractional extension of the Monodomain model to account for both fibres anisotropies and tissue micro-heterogeneities in the modelling and numerical simulation of cardiac electrophysiology. The numerical results we presented here show the characteristic

Fibers direction

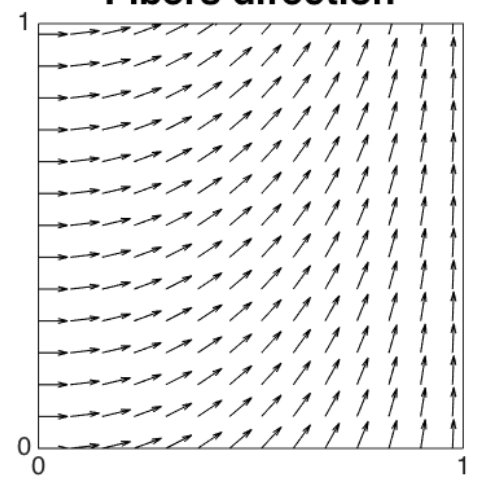

Curve A

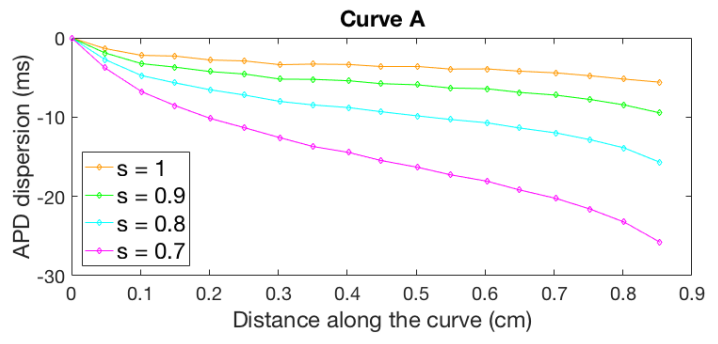

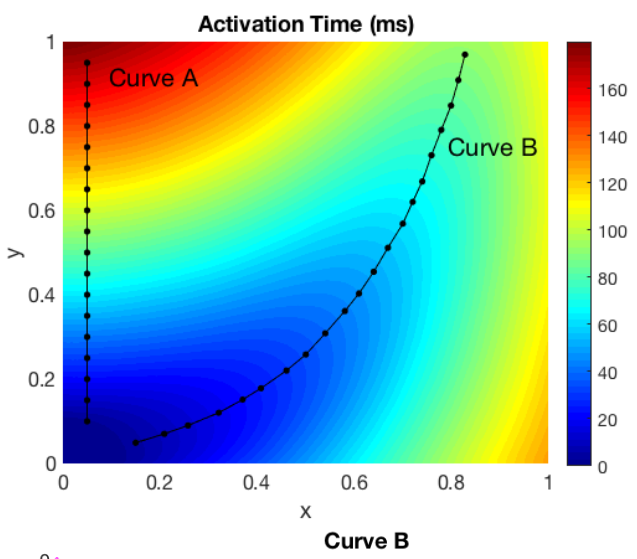

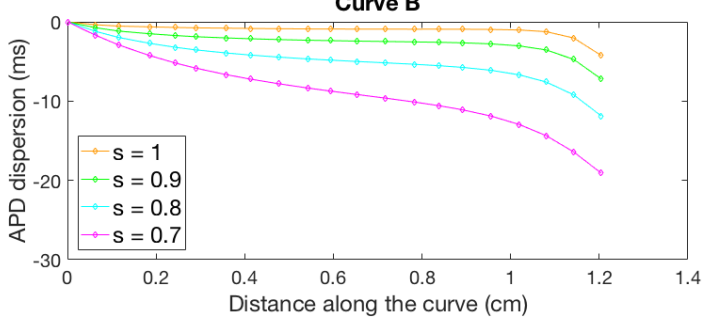

Figure 8. Test 3. Anisotropic tissue with fibres rotation. Top: fibres rotation (left) and activation time (right). Bottom: APD dispersion along the curves A and $\mathrm{B}$. 
features of both aspects. Fibers directions trigger anisotropic propagation patterns, as in the case of the classical Monodomain and Bidomain models. In addition, powers of the diffusion operator in the Fractional Monodomain model result in behaviours that are physically expected in the presence
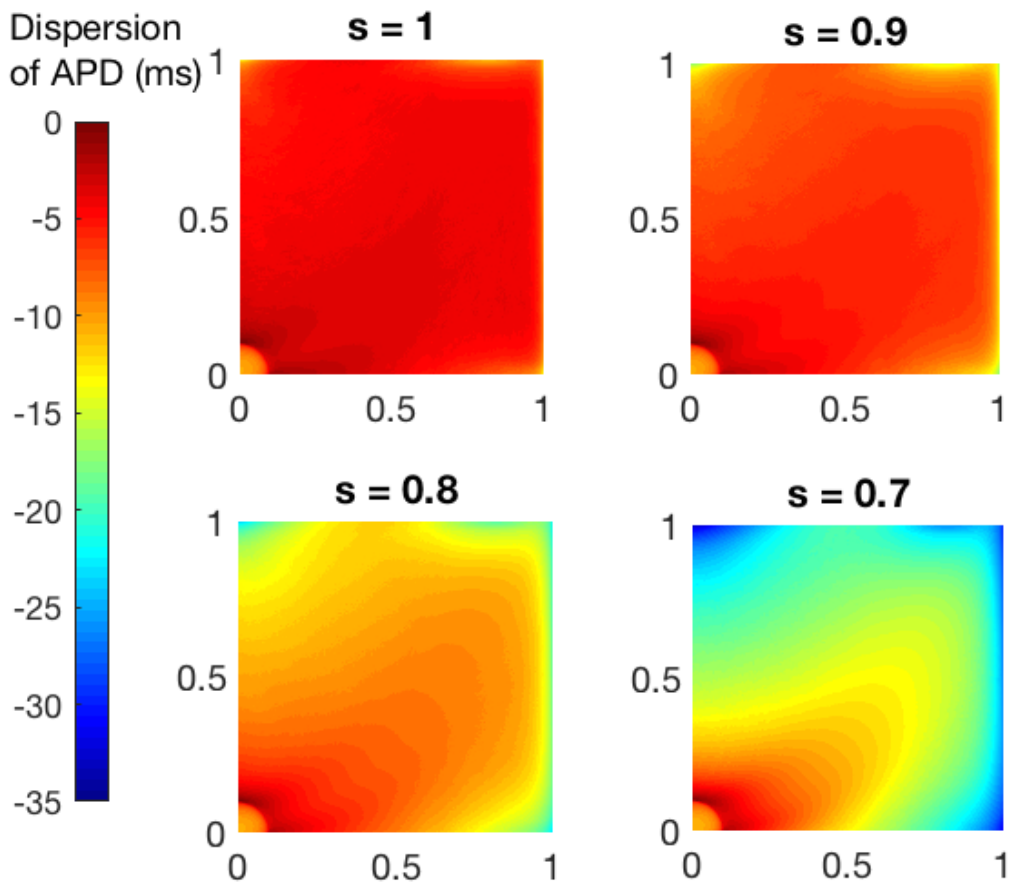

Figure 9. Test 3. Anisotropic tissue with fibres rotation. APD dispersion on $\Omega$ for the considered values of $s$.

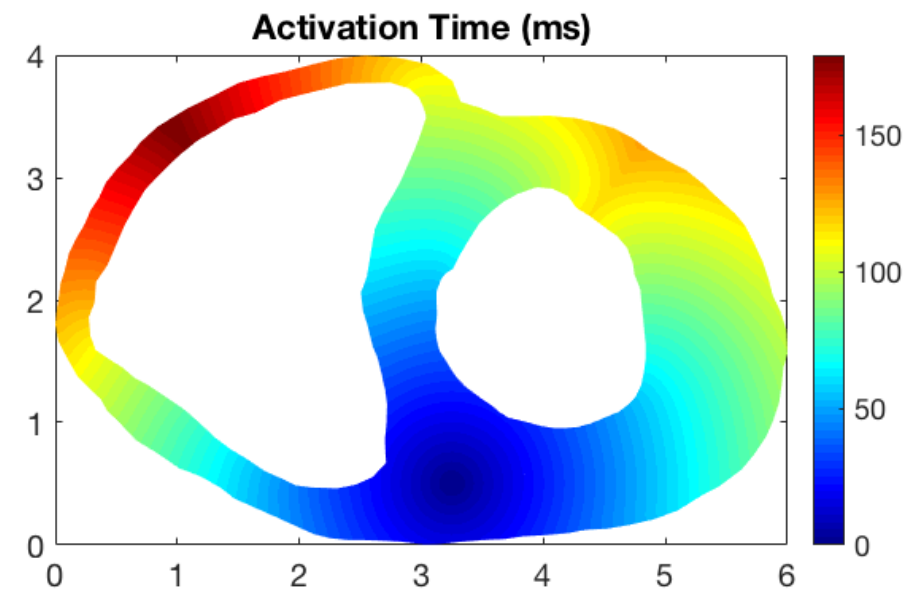

Figure 10. Test 4. Activation time on the cardiac slice. Spatial units are in $\mathrm{cm}$. 

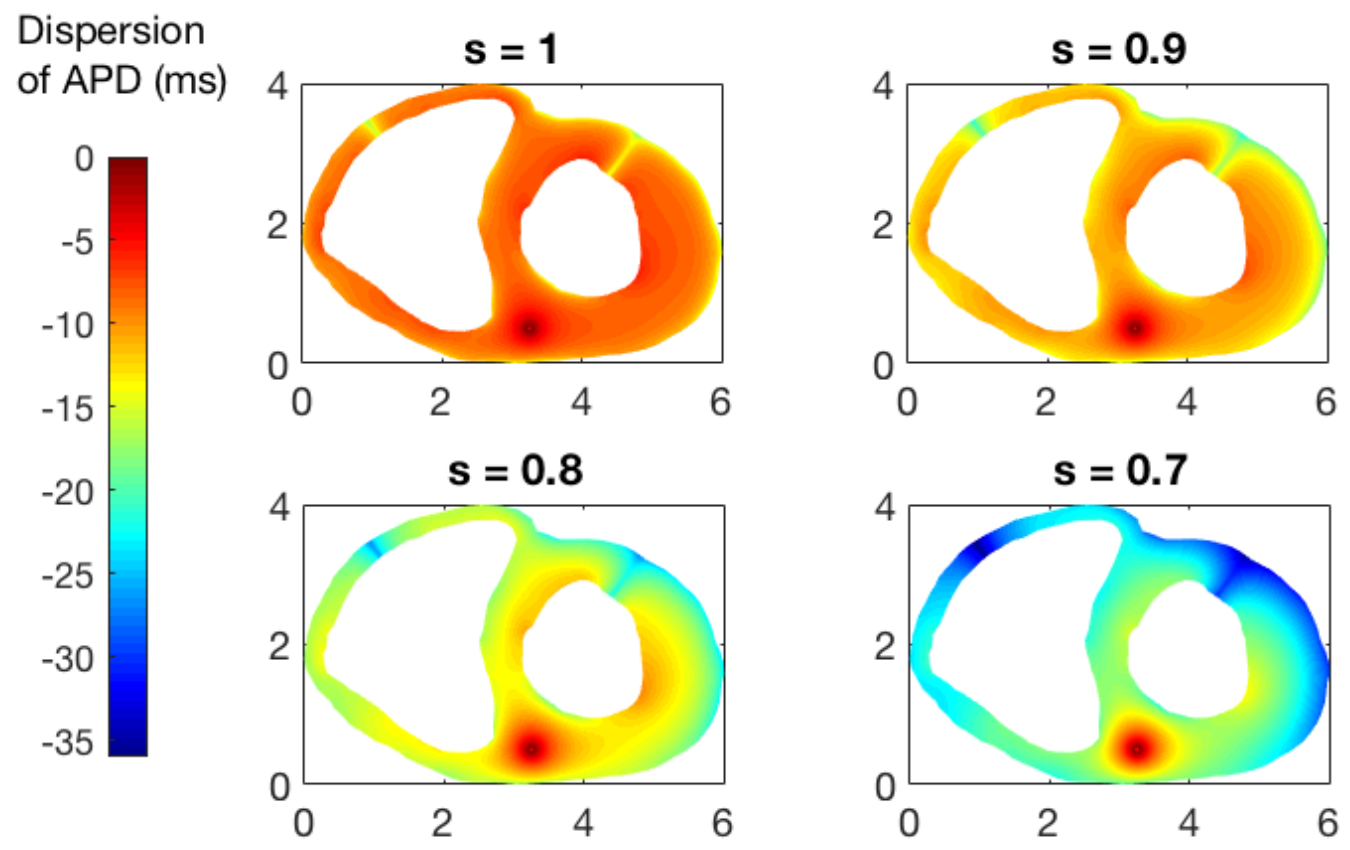

Figure 11. Test 4. APD dispersion on the cardiac slice for the considered values of $s$.

of structural micro-heterogeneities of the tissue, such as a wider action potential foot, a lower peak of the membrane voltage during an action potential, and an evident dispersion of the Action Potential Duration as the excitation wave moves away from the stimulus source. As expected from the theory of fractional operators, these effects get more pronounced ad the fractional exponent $s \in(0,1)$ moves away from 1 , the homogeneous case. These results are extremely promising, and we will validate the model against experimental data. Still, further work needs to be done to improve the computational efficiency of the numerical solution. The main bottleneck in using a space-fractional operator is the need of very fine spatial grids (much finer than in the classical case) due to the fact that AP shape changes with the fractional order. In particular, as $s$ is reduced, the model produces APs that display a sharper peak. As it was already observed in the one-dimensional case in [18], this is an intrinsic feature of the Fractional Monodomain and it does not depend on the conduction velocity. The higher spatial resolution required to accurately capture the travelling pulse of the fractional case is thus unavoidable (at least in proximity of the propagating front). In addition, the numerical simulation of the Fractional Monodomain requires higher computational times than that of the classical Monodomain. In this paper we used the Contour Integral method (CI) from [17]: it is effective but intrinsically slow, although parallel. We are currently investigating more efficient ways to compute the matrix power $A^{s}$, and we plan to compare the efficiency of the CI with an explicit scheme based on the approximation of the spectral Fractional Laplacian introduced in [16]. Finally, the method introduced here associates a given value of $s$ to the entire spatial domain. This amounts to implicitly assume a uniform level of micro-heterogeneity across the whole tissue. This is a limiting assumption, that we plan to overcome in a forthcoming paper. 


\section{ACKNOWLEDGEMENTS}

This work was supported by the Basque Government through the BERC 2014-2017 program, and by the Spanish Ministry of Economics and Competitiveness MINECO through the BCAM Severo Ochoa excellence accreditation SEV-2013-0323 and the Spanish "Plan Estatal de Investigación, Desarrollo e Innovación Orientada a los Retos de la Sociedad" under Grant BELEMET - Brain ELEctro-METabolic modeling and numerical approximation (MTM2015-69992-R).

\section{REFERENCES}

[1] F. Sachse, Computational Cardiology, Springer, Berlin, 2004.

[2] A. Azzouzi, Y. Coudière, R. Turpault, N. Zemzemi. A mathematical model of the Purkinje-muscle junctions. Math. Biosci. Eng., 8 (4) (2011), pp. 915-930.

[3] C. Vergara, M. Lange, S. Palamara, T. Lassila, A.F. Frangi, A. Quarteroni, A coupled 3D-1D numerical monodomain solver for cardiac electrical activation in the myocardium with detailed Purkinje network, Journal of Computational Physics, Vol. 308, 2016, Pages 218-238.

[4] M.J Bishop and G. Plank, The role of fine-scale anatomical structure in the dynamics of reentry in computational models of the rabbit ventricles. J Physiol 590 (2012), p. 4515-4535.

[5] A. Bueno-Orovio, D. Kay, V. Grau, B. Rodriguez, K. Burrage, Fractional diffusion models of cardiac electrical propagation: role of structural heterogeneity in dispersion of repolarization, J. R. Soc. Interface 11 (2014) 20140352.

[6] M. S. Spach, R. C. Barr, Effects of cardiac microstructure on propagating electrical waveforms, Circ. Res. 86 (2000) e23-e28.

[7] B. Hanson, P. Sutton, N. Elameri, M. Gray, H. Critchley, J. S. Gill, P. Taggart, Interaction of activationrepolarization coupling and restitution properties in humans, Circ. Arrhythm. Electrophysiol. 2 (2) (2009) 162170.

[8] N. Mercadier, W. Guerin, M. Chevrollier, and R. Kaiser, Lévy flights of photons in hot atomic vapours, Nature Phys., 5 (2009), pp. 602-605.

[9] R. Metzler, J.-H. Jeon, A. G. Cherstvy, and E. Barkai, Anomalous diffusion models and their properties: nonstationarity, non-ergodicity, and ageing at the centenary of single particle tracking, Phys. Chem. Chem. Phys., 16 (2014), p. 24128.

[10] S. Ratynskaia, K. Rypdal, C. Knapek, S. Khrapak, A. V. Milovanov, A. Ivlev, J. J. Rasmussen, and G. E. Morfill, Superdiffusion and viscoelastic vortex flows in a two-dimensional complex plasma, Phys. Rev. Lett., 96 (2006), p. 105010.

[11] Y. A. Rossikhin and M. V. Shitikova, Application of fractional calculus for dynamic problems of solid mechanics: novel trends and recent results, Appl. Mech. Rev., 63 (2010), p. 010801.

[12] Y. Zhang, X. Liu, M. R. Belić, W. Zhong, Y. Zhang, and M. Xiao, Propagation dynamics of a light beam in a fractional Schrödinger equation, Phys. Rev. Lett., 115 (2015), p. 180403.

[13] Y. Zhang, M. M. Meerschaert, and R. M. Neupauer, Backward fractional advection dispersion model for contaminant source prediction, Water Resour. Res., 52 (2016), pp. 2462-2473.

[14] A. Bueno-Orovio, I. Teh, J. Schneider, K. Burrage, V. Grau Anomalous diffusion in cardiac tissue as an index of myocardial microstructure, IEEE Trans. Me. Imaging 35 (2016) 2200-2207.

[15] F. Song, C. Xu, and G.E. Karniadakis. Computing fractional Laplacians on complex-geometry domains: algorithms and simulations. SIAM J. Sci. Comp., 39 (4), pp. A1320-A1344, 2017.

[16] N. Cusimano, F. del Teso, L. Gerardo-Giorda, G. Pagnini, Discretisations of the fractional laplacian on general domains with Dirichlet, Neumann, and Robin boundary conditions, in revision (2017), arXiv:1708.03602.

[17] K. Burrage, N. Hale, D. Kay, An efficient implicit FEM scheme for fractional-in-space diffusion equations, SIAM J. Sci. Comput. 34 (4) (2012) A2145-A2172.

[18] N. Cusimano, A. Bueno-Orovio, I. Turner, K. Burrage, On the order of the fractional laplacian in determining the spatio-temporal evolution of a space-fractional model of cardiac electrophysiology, PLoS ONE 10 (2015) e0143938.

[19] E.J. Vigmond, F. Aguel, and N.A. Trayanova. Computational techniques for solving the bidomain equations in three dimensions. IEEE Trans. Biomed. Eng., 49(11):1260-1269, 2002.

[20] E.J. Vigmond, R. Weber dos Santos, A.J. Prassl, M. Deo, and G. Plank. Solvers for the caridac bidomain equations. Progress in Biophysics and Molecular Biology, 96(1-3):3-18, 2008.

[21] M. Pennacchio and V. Simoncini. Efficient algebraic solution of rection-diffusion systems for the cardiac excitation process. J. Comput. Appl. Math., 145(1):49-70, 2002. 
[22] R.H. Clayton and A.V. Panfilov. A guide to modelling cardiac electrical activity in anatomically detailed ventricles. Progress in Biophysics and Molecular Biology, 96(1-3):19-43, 2008.

[23] P. Colli Franzone and L.F. Pavarino. A parallel solver for reaction-diffusion systems in computational electrocardiology. Mathematical models and methods in applied sciences, 14(6):883-911, 2004.

[24] L. Gerardo-Giorda and M. Perego, Optimized Schwarz Methods for the Bidomain system in electrocardiology, M2AN, Vol. 47 (2), pp 583-608, 2013.

[25] R. Weber dos Santos, G. Planck, S. Bauer, and E.J. Vigmond. Parallel multigrid preconditioner for the cardiac bidomain model. IEEE Trans. Biomed. Eng., 51(11):1960-1968, 2004.

[26] L.F. Pavarino and S. Scacchi. Multilevel additive Schwarz preconditioners for the Bidomain reaction-diffusion system. SIAM J. Sci. Comp., 31(1):420-443, 2008.

[27] S. Scacchi. A hybrid multilevel Schwarz method for the bidomain model. Computer Methods in Applied Mechanics and Engineering, 197(45-48):4051-4061, August 2008.

[28] L. Gerardo-Giorda, L. Mirabella, F. Nobile, M. Perego, and A. Veneziani. A model-based block-triangular preconditioner for the Bidomain system in electrocardiology. J. Comp. Phys., 228:3625-3639, 2009.

[29] M. Potse, B. Dubé, J. Richer, and A. Vinet, A comparison of Monodomain and Bidomain Reaction-Diffusion models for Action Potential Propagation in the Human Heart. IEEE Trans. Biomed. Eng., 53(12):2425-2435, 2006.

[30] P. Colli Franzone, L.F. Pavarino, and B. Taccardi. Simulating patterns of excitation, repolarization and action potential duration with cardiac Bidomain and Monodomain models. Math. Biosc., 197:35-66, 2005.

[31] Prakosa A, Malamas P, Zhang S, Pashakhanloo F, Arevalo H, Herzka DH, Lardo A, Halperin H, McVeigh E, Trayanova N, Vadakkumpadan F. Methodology for image-based reconstruction of ventricular geometry for patient-specific modeling of cardiac electrophysiology, Progress in biophysics and molecular biology 2014; 115(23): 226-234, doi: 10.1016/j.pbiomolbio.2014.08.009.

[32] Sermesant M, Chabiniok R, Chinchapatnam P, Mansi T, Billet F, Moireau P, Peyrat JM, Wong K, Relan J, Rhode K, Ginks M, Lambiase P, Delingette H, Sorine M, Rinaldi CA, Chapelle D, Razavi R, Ayache N. Patientspecific electromechanical models of the heart for the prediction of pacing acute effects in CRT: A preliminary clinical validation, Medical image analysis 2012, Elsevier, 16(1), pp. 201-215, doi: 10.1016/j.media.2011.07.003.

[33] E. Ukwatta, H. Arevalo, M. Rajchl, J. White, F. Pashakhanloo A. Prakosa, D.A Herzka DA, E. McVeigh, A.C. Lardo, N. Trayanova, F. Vadakkumpadan. Image-based reconstruction of three-dimensional myocardial infarct geometry for patient-specific modeling of cardiac electrophysiology, Medical physics 2015; 42(8): 4579-4590, doi: $10.1118 / 1.4926428$.

[34] F. Vadakkumpadan, L.J. Rantner, B. Tice, P. Boyle, A.J. Prassl, E. Vigmond, G. Plank, N. Trayanova. Image-based models of cardiac structure with applications in arrhythmia and defibrillation studies, Journal of Electrocardiology 2009; 2(42): 157.e1-10, doi: 10.1016/j.jelectrocard.2008.12.003.

[35] J. Le Grice, B.H. Smaill, L.Z. Chai, S.G. Edgar, J.B. Gavin, and P.J. Hunter. Laminar structure of the heart: ventricular myocyte arrangement and connective tissue architecture in the dog. Am. J. Physiol., 269 (Heart Circ. Physiol.)(38):H571-H582, 1995.

[36] D. Streeter. Gross morphology and fiber geometry in the heart. In R.M. Berne, editor, Handbook of Physiology, volume 1 (Sec. 2), pages 61-112. Williams and Wilnkins, 1979.

[37] G.W. Beeler and H. Reuter, Reconstruction of the action potential of ventricular myocardial fibres. J. Physiol. 268 (1977), p. 177-210.

[38] P. Stinga, Fractional powers of second order partial differential operators: extension problem and regularity theory, PhD thesis, Universidad Autónoma de Madrid, 2010.

[39] J. P. Whiteley, An efficient numerical technique for the solution of the Monodomain and bidomain equations, IEEE Trans. Biomed. Eng., 53:21392147, 2006.

[40] N. Hale, N. J. Higham, L. N. Trefethen, Computing $A^{\alpha}, \log (A)$, and related matrix functions by contour integrals, SIAM J. Numer. Anal. 46 (5) (2008) 2505-2523.

[41] A. Quarteroni, R. Sacco, F. Saleri, Numerical Mathematics, Springer New York-Berlin-Heidelberg, 2000.

[42] T. A. Driscoll, The Schwarz-Christoffel toolbox, available online at http://www.math.udel.edu/ driscoll/SC/

[43] M. Sreenivas. A study of incidence and severity of coronary artery stenosis at autopsy Anil Aggrawal's Internet Journal of Forensic Medicine and Toxicology, 2009; Vol. 10, No. 1.

Basque Center for Applied Mathematics, Bilbao, Spain, ncusimano@bcamath.org

Basque Center for Applied Mathematics, Bilbao, Spain, lgerardo@bcamath.org 